Supporting Materials to

\title{
Palladium-Catalyzed Cross-coupling Reaction of Carboxylic Anhydrides with Organozinc Reagents
}

Donghui Wang and Zhaoguo Zhang*

State Key Laboratory of Organometallic Chemistry, Shanghai Institute of Organic Chemistry, Shanghai 200032, China

Experimental section.............................................. 3

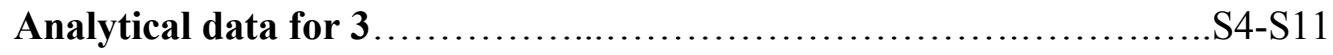

References.......................................................... 113 


\section{Experimental Section}

All reactions were carried out under argon atmosphere using standard Schlenk techniques. Solvents were purified by the standard methods. ${ }^{1} \mathrm{Ph}_{2} \mathrm{Zn}$ was commercially available from Acros. The carboxylic anhydrides were prepared according to literature procedure. ${ }^{2} \mathrm{Pd}\left(\mathrm{PPh}_{3}\right)_{4}$ was prepared according to literature procedure. ${ }^{3}{ }^{1} \mathrm{HNMR}$ (300 $\mathrm{MHz}),{ }^{13} \mathrm{CNMR}(75.4 \mathrm{MHz})$ and ${ }^{19} \mathrm{FNMR}(282 \mathrm{MHz})$ were registered with $\mathrm{CDCl}_{3}$ as solvent and tetramethylsilane (TMS) as internal standard. Chemical shifts are reported in units (ppm) by assigning TMS resonance in the ${ }^{1} \mathrm{H}$ spectrum as $0.00 \mathrm{ppm}$ and $\mathrm{CDCl}_{3}$ resonance in the ${ }^{13} \mathrm{C}$ spectrum as $77.0 \mathrm{ppm}$. All coupling constants ( $J$ values) were reported in Hertz (Hz). Column chromatography was performed on silica gel 300-400 mesh. Gas chromatographic analysis (GC) was carried out on an Angilent-6890N equipped with capillary column $\left(0.25 \mathrm{~mm}\right.$ i.d.X30 m), using $\mathrm{N}_{2}$ as carrier gas. Analytical thin-layer chromatography (TLC) was visualized with UV light (254 and $365 \mathrm{~nm}$ ) or by iodine vapor staining. Electron impact (EI, $70 \mathrm{eV}$ ) was used as the ionization method for the mass spectrometry (percent values were given in parentheses). Mass data were reported in mass units $(\mathrm{m} / \mathrm{z})$.

General Procedure For the Palladium-Catalyzed Reaction of Carboxylic Anhydrides and Organozinc Reagents (Table 1). A typical procedure is as follows: To a THF solution $(3 \mathrm{~mL})$ containing benzoic anhydride $(227 \mathrm{mg}, 1.00 \mathrm{mmol})$ in a dry 25 mL Schlenk tube under argon, $\mathrm{Ph}_{2} \mathrm{Zn}\left(286 \mathrm{mg}, 1.2 \mathrm{mmol}, 92 \%\right.$ purity) and $\mathrm{Pd}\left(\mathrm{PPh}_{3}\right)_{4}(58$ $\mathrm{mg}, 0.05 \mathrm{mmol}$ ) were added at room temperature. The reaction mixture was then heated in an oil bath $\left(80^{\circ} \mathrm{C}\right)$ for 18 hours. The yield of benzophenone was determined by $\mathrm{GC}$ using dibenzyl ether as an internal standard and characterized by comparison with an authentic sample.

\section{Cross-Coupling Reaction of Carboxylic Anhydrides With Organozinc Reagents} in the Presence of $\mathbf{P d}\left(\mathrm{PPh}_{3}\right)_{4}$ (Table 2). A typical procedure is as follows: To the THF solution $(3 \mathrm{~mL})$ containing $p$-toluic anhydride $(240 \mathrm{mg}, 1.00 \mathrm{mmol})$ in a dry $25 \mathrm{ml}$ Schlenck tube under argon, $\mathrm{Ph}_{2} \mathrm{Zn}\left(286 \mathrm{mg}, 1.2 \mathrm{mmol}, 92 \%\right.$ purity) and $\mathrm{Pd}\left(\mathrm{PPh}_{3}\right)_{4}(58 \mathrm{mg}$, $0.05 \mathrm{mmol}$ ) were added at room temperature. The system was then heated under argon in an oil bath $\left(80^{\circ} \mathrm{C}\right)$ for 18 hours. After cooling the reaction mixture, diethyl ether $(50 \mathrm{~mL})$ and $\mathrm{H}_{2} \mathrm{O}(50 \mathrm{~mL})$ were added and the aqueous layer was extracted with diethyl ether(50 
mLX3). The combined ether solution was dried $\mathrm{Na}_{2} \mathrm{SO}_{4}$, filtered and then evaporated in vacuum. Purification of the residue by column chromatography gave the corresponding product 3b (138 mg; yield 70\%).

Coupling of $\mathbf{P h}_{2} \mathrm{Zn}$ with Mixed Anhydride in situ (Table 3). A typical procedure is as follows (entry 1): To a mixture of benzoic acid (122 $\mathrm{mg}, 1 \mathrm{mmol})$ and $\mathrm{Et}_{3} \mathrm{~N}(0.14$ $\mathrm{mL}, 1 \mathrm{mmol})$ under argon in ice- salt bath, ethyl chloroformate $(0.095 \mathrm{~mL}, 1 \mathrm{mmol})$ was added. Then the system was stirred at room temperature for 15 minutes. Finally, $\mathrm{Ph}_{2} \mathrm{Zn}$ (286 $\mathrm{mg}, 1.2 \mathrm{mmol}, 92 \%$ purity) and $\mathrm{Pd}\left(\mathrm{PPh}_{3}\right)_{4}(58 \mathrm{mg}, 0.05 \mathrm{mmol})$ were added and the reaction mixture was heated in an oil bath $\left(80^{\circ} \mathrm{C}\right)$ for 16 hours. The yield of benzophenone was determined by GC using dibenzyl ether as an internal standard and characterized by comparison with an authentic sample.

Synthesis of Ketones from Sodium Carboxylates and Organozinc Reagents. A typical procedure is as follows: To a mixture of sodium benzoate $(288 \mathrm{mg}, 2 \mathrm{mmol})$ and ethyl chloroformate $(0.57 \mathrm{~mL}, 6 \mathrm{mmol})$ in dry THF $(5 \mathrm{~mL})$ that had been stirred for 15 min at room temperature under argon, $\mathrm{Ph}_{2} \mathrm{Zn}(572 \mathrm{mg}, 2.4 \mathrm{mmol}, 92 \%$ purity) and $\mathrm{Pd}\left(\mathrm{PPh}_{3}\right)_{4}(116 \mathrm{mg}, 0.10 \mathrm{mmol})$ were added. Then the system temperature was raised to $70^{\circ} \mathrm{C}$ and kept at this temperature for 14 hours. After cooling the reaction mixture, diethyl ether $(50 \mathrm{~mL})$ and $\mathrm{H}_{2} \mathrm{O}(50 \mathrm{~mL})$ were added and the aqueous layer was extracted with diethyl ether(50 mL X 3). The combined ether solution was dried $\left(\mathrm{Na}_{2} \mathrm{SO}_{4}\right)$, filtered and evaporated in vacuum. Purification of the residue by column chromatography gave the corresponding product 3a (346 mg; yield 95\%).

\section{Analytical data for $3 b-3 q$}




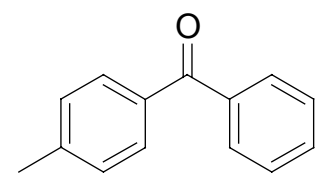

$3 b$

4-Methylbenzophenone (3b) ${ }^{4}:{ }^{1} \mathrm{H}$ NMR $\left(300 \mathrm{MHz}, \mathrm{CDCl}_{3}\right): \delta 7.78(\mathrm{~d}, J=7.0 \mathrm{~Hz}, 2 \mathrm{H})$, $7.72(\mathrm{~d}, J=7.0 \mathrm{~Hz}, 2 \mathrm{H}), 7.57$ (t, $J=7.0 \mathrm{~Hz}, 1 \mathrm{H}), 7.47$ (t, $J=7.0 \mathrm{~Hz}, 2 \mathrm{H}), 7.28$ (d, 2H, $J$ $=7.0 \mathrm{~Hz}), 2.44(\mathrm{~s}, 3 \mathrm{H}) ;{ }^{13} \mathrm{C} \mathrm{NMR}\left(75 \mathrm{MHz}, \mathrm{CDCl}_{3}\right): \delta 196.5,143.2,137.9,134.8,132.2$, 130.3, 129.9, 128.9, 128.2, 21.7; MS (EI) (\%): $196\left(\mathrm{M}^{+}, 43\right), 181(9), 165(4), 152(4)$, 19(100), 105(32), 91(46), 77(43); IR: 1656, 1606, 1317, 1278, 836, 730, $700 \mathrm{~cm}^{-1}$.

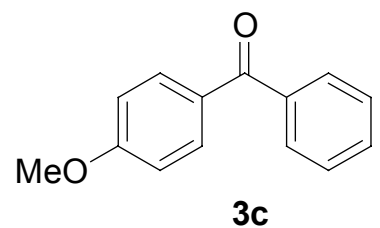

4-Methoxybenzophenone (3c) $)^{5}:{ }^{1} \mathrm{H}$ NMR (300 MHz, $\left.\mathrm{CDCl}_{3}\right)$ : $\delta 7.82(\mathrm{~m}, 2 \mathrm{H}), 7.76-$ $7.73(\mathrm{~m}, 2 \mathrm{H}), 7.55(\mathrm{t}, J=7.0 \mathrm{~Hz}, 1 \mathrm{H}), 7.46(\mathrm{t}, J=7.0 \mathrm{~Hz}, 2 \mathrm{H}), 6.95(\mathrm{~m}, 2 \mathrm{H}), 3.87(\mathrm{~s}, 3 \mathrm{H})$; ${ }^{13} \mathrm{C}$ NMR $\left(75 \mathrm{MHz}, \mathrm{CDCl}_{3}\right)$ : $\delta \quad 197.2,163.2,138.3,132.6,131.9,130.1,129.7,128.2$, 113.5, 55.5; MS (EI): 212(M+ 436), 181 (3), 135 (100), 105 (10), 92 (12), 77 (28), 51 (6); IR (KBr) 1653, 1602, 1510, 1171, 1030, 846, 742, $702 \mathrm{~cm}^{-1}$.<smiles>O=C(c1ccccc1)c1ccc(C(F)(F)F)cc1</smiles>

4-Trifluoromethylbenzophenone (3d) ${ }^{6}:{ }^{1} \mathrm{H}$ NMR $\left(300 \mathrm{MHz}, \mathrm{CDCl}_{3}\right): \quad \delta 7.51(\mathrm{~d}, J=7.2$ $\mathrm{Hz}, 2 \mathrm{H}), 7.63(\mathrm{t}, J=7.2 \mathrm{~Hz}, 1 \mathrm{H}), 7.76(\mathrm{~d}, J=8.1 \mathrm{~Hz}, 2 \mathrm{H}), 7.81(\mathrm{~d}, J=8.1 \mathrm{~Hz}, 2 \mathrm{H}), 7.90$ $(\mathrm{d}, J=7.2 \mathrm{~Hz}, 2 \mathrm{H}) ;{ }^{13} \mathrm{C} \mathrm{NMR}\left(75 \mathrm{MHz}, \mathrm{CDCl}_{3}\right): \delta 195.5,140.7$ (q, $\left.J=4.2 \mathrm{~Hz}\right), 136.7$, 
$133.9,133.5,133.0,130.0(\mathrm{~d}, J=10.4 \mathrm{~Hz}), 128.5,125.3(\mathrm{q}, J=14.1 \mathrm{~Hz}), 121.8 ;{ }^{19} \mathrm{~F}$ $\operatorname{NMR}\left(282 \mathrm{MHz}, \mathrm{CDCl}_{3}\right): \delta-63.4$.

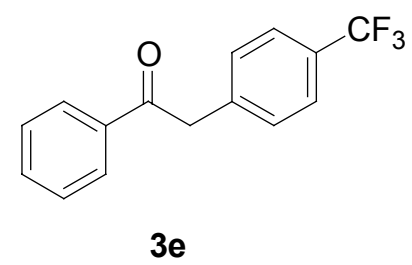

4'-Trifluoromethyl-deoxybenzoin (3e): ${ }^{1} \mathrm{H}$ NMR (300 MHz, $\left.\mathrm{CDCl}_{3}\right)^{7}: \delta 4.35(\mathrm{~s}, 2 \mathrm{H})$, $7.38(\mathrm{~d}, J=8.4 \mathrm{~Hz}, 2 \mathrm{H}), 7.43-7.53(\mathrm{~m}, 2 \mathrm{H}), 7.55-7.62(\mathrm{~m}, 2 \mathrm{H}), 7.70-7.81(\mathrm{~m}, 1 \mathrm{H}), 7.99-$ $8.02(\mathrm{~m}, 2 \mathrm{H}) ;{ }^{13} \mathrm{C} \mathrm{NMR}\left(75 \mathrm{MHz}, \mathrm{CDCl}_{3}\right)$ : $\delta$ 196.7, 142.8, 138.5, 136.3, 135.3, 133.5, 139.1, 128.8, 128.5, 125.5 (q, $J=15.2 \mathrm{~Hz}), 44.99 ;{ }^{19} \mathrm{~F}$ NMR $\left(282 \mathrm{MHz} \mathrm{CDCl}_{3}\right): \delta$-62.9;

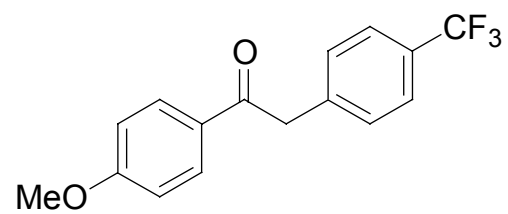

$3 \mathbf{f}$

1-(4-methoxy-phenyl)-2-(4-trifluoromethyl-phenyl)-ethanone (3f) ${ }^{8}:{ }^{1} \mathrm{H}$ NMR (300 MHz, $\left.\mathrm{CDCl}_{3}\right): \delta 3.87(\mathrm{~s}, 3 \mathrm{H}), 4.30(\mathrm{~s}, 2 \mathrm{H}), 6.95(\mathrm{~d}, J=9 \mathrm{~Hz}, 2 \mathrm{H}), 7.38(\mathrm{~d}, J=8 \mathrm{~Hz}, 2 \mathrm{H}), 7.58$ (d, $J=8 \mathrm{~Hz}, 2 \mathrm{H}), 7.99(\mathrm{~d}, J=9 \mathrm{~Hz}, 2 \mathrm{H}) ;{ }^{13} \mathrm{C} \mathrm{NMR}\left(75 \mathrm{MHz}, \mathrm{CDCl}_{3}\right)$ : $\delta$ 195.2, 163.8, $138.9(\mathrm{q}, J=3.4 \mathrm{~Hz}), 130.8,129.9,129.9,129.3,128.9,125.5$ (q, $J=13.8 \mathrm{~Hz}), 113.9$, 55.5, 44.8; ${ }^{19} \mathrm{~F}$ NMR (282 MHz, $\left.\mathrm{CDCl}_{3}\right): \delta$-62.9;<smiles>O=C(Cc1ccc(C(F)(F)F)cc1)c1ccc(C(F)(F)F)cc1</smiles>

$3 g$ 
1,2-bis-(4-trifluoromethyl-phenyl)-ethanone (3g) $)^{9}:{ }^{1} \mathrm{H} \mathrm{NMR}\left(300 \mathrm{MHz}, \mathrm{CDCl}_{3}\right): \delta 4.38$ (s, 2H), 7.38 (d, $J=8.0 \mathrm{~Hz}, 2 \mathrm{H}), 7.60$ (d, $J=8.0 \mathrm{~Hz}, 2 \mathrm{H}), 7.75$ (d, $J=8.0 \mathrm{~Hz}, 2 \mathrm{H}), 8.11$ $(\mathrm{d}, J=8.0 \mathrm{~Hz}, 2 \mathrm{H}) ;{ }^{13} \mathrm{C} \mathrm{NMR}\left(75 \mathrm{MHz}, \mathrm{CDCl}_{3}\right): \delta 196.6,166.1,158.5,142.4,138.9$ (q, $J=4.0 \mathrm{~Hz}), 137.0$ (q, $J=5.4 \mathrm{~Hz}), 129.9,128.8,125.9$ (q, $J=14.1 \mathrm{~Hz}), 125.7$ (q, $J=13.5$ $\mathrm{Hz}), 122.3,45.5 ;{ }^{19} \mathrm{~F}$ NMR (282 MHz, $\left.\mathrm{CDCl}_{3}\right): \delta-63.02,-63.6$.

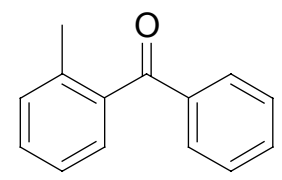

$3 h$

2-Methylbenzophenone (3h) ${ }^{10}:{ }^{1} \mathrm{H}$ NMR (300 MHz, $\left.\mathrm{CDCl}_{3}\right): \delta \quad 2.33$ (s, 3H), 7.20-7.32 (m, 3H), 7.35-7.46 (m, 3H), 7.53-7.58 (m, 1H), 7.78-7.81 (m, 2H); ${ }^{13} \mathrm{C}$ NMR (75 MHz, $\left.\mathrm{CDCl}_{3}\right): \delta \quad 198.5,138.5,137.6,136.6,133.0,130.9,130.1,130.0,128.4,128.4,125.1$, 19.9; IR (KBr): 1666, 1598, 1449, 1315, 1268, 925, 732, $709 \mathrm{~cm}^{-1}$; MS (EI): 196(M+( 67), 195 (100), 178 (11), 119 (29), 105 (21), 91 (33), 77(33), 51 (13).

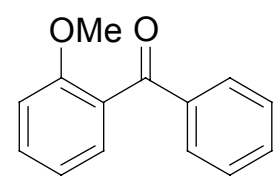

$3 \mathbf{i}$

2-Methoxybenzophenone (3i) $)^{11}:{ }^{1} \mathrm{H}$ NMR $\left(300 \mathrm{MHz} \mathrm{CDCl}_{3}\right): \delta 3.72(\mathrm{~s}, 3 \mathrm{H}), 6.98-7.07$ $(\mathrm{m}, 2 \mathrm{H}), 7.35-7.50(\mathrm{~m}, 4 \mathrm{H}), 7.52-7.58(\mathrm{~m}, 1 \mathrm{H}), 7.79-7.84(\mathrm{~m}, 2 \mathrm{H}) ;{ }^{13} \mathrm{C} \mathrm{NMR}(75 \mathrm{MHz}$, $\left.\mathrm{CDCl}_{3}\right): \delta 55.5,111.4,120.4,128.2,128.7,129.5,129.8,131.8,132.9,137.7 .157 .3$, 196.5; MS (EI): 213 (M+1 $\left.{ }^{+}, 15.61\right), 212\left(\mathrm{M}^{+}, 35\right), 195$ (20), 135 (80), 121 (22), 105 (39), 77 (100), 51 (46); IR (KBr): 1666, 1599, 1488, 1245, 1024, 926, 755, $703 \mathrm{~cm}^{-1}$. 


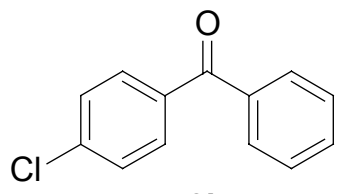

3j

4-Chlorobenzophenone (3j) $)^{12}:{ }^{1} \mathrm{H}$ NMR $\left(300 \mathrm{MHz}, \mathrm{CDCl}_{3}\right): \quad \delta \quad$ 7.78-7.73 (m, 4H), 7.677.57 (m, 1H), 7.51-7.44 (m, 4H); ${ }^{13} \mathrm{C}$ NMR: (75 MHz, $\left.\mathrm{CDCl}_{3}\right)$ : $\delta \quad 195.5$ 138.8, 137.2, 135.8, 132.6, 131.4, 129.9, 128.6, 128.4; MS (EI): $218\left(\mathrm{M}^{+},{ }^{37} \mathrm{Cl}, 8\right), 216\left(\mathrm{M}^{+},{ }^{35} \mathrm{Cl}, 24\right)$, 181 (10), 139 (52), 111 (52), 105 (83), 77 (100), 51 (72); IR (KBr) 1651, 1584, 1281, $1089,844,728,695 \mathrm{~cm}^{-1}$.

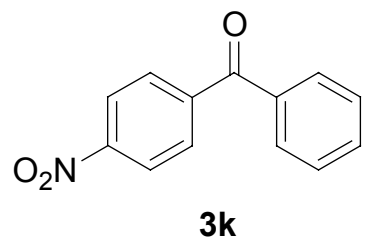

4-Nitrobenzophenone (3k) $)^{13}:{ }^{1} \mathrm{H}$ NMR $\left(300 \mathrm{MHz}, \mathrm{CDCl}_{3}\right): \delta 7.53$ (t, $\left.J=7.8 \mathrm{~Hz}, 2 \mathrm{H}\right)$, 7.66 (t, $J=7.8 \mathrm{~Hz}, 1 \mathrm{H}), 7.80$ (d, $J=7.8 \mathrm{~Hz}, 2 \mathrm{H}), 7.94$ (d, $J=8.6 \mathrm{~Hz}, 2 \mathrm{H}), 8.32-37$ (d, $J=$ $8.6 \mathrm{~Hz}, 2 \mathrm{H}) ;{ }^{13} \mathrm{C}$ NMR: $\left(75 \mathrm{MHz}, \mathrm{CDCl}_{3}\right): \delta$ 194.8, 142.8, 136.2, 133.4, 130.6, 130.1, 128.7,128.6 123.5; MS (EI): $227\left(\mathrm{M}^{+}, 53\right)$, 181, (4), 150 (13), 105 (100), 77 (578), 76 (19), 51 (20); IR (KBr): 3101, 1651, 1595, 1514, 1359, 1318, 1106, 930, 873, 734, 705 $\mathrm{cm}^{-1}$.

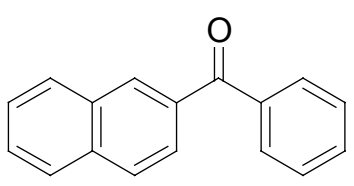

3I

2-Benzoylnaphthane (3I) $)^{14}:{ }^{1} \mathrm{H}$ NMR $\left(300 \mathrm{MHz}, \mathrm{CDCl}_{3}\right): \quad \delta \quad 7.49-7.65(\mathrm{~m}, 5 \mathrm{H}), 7.84-$ 7.95(m, 6H), 8.26 (s, 1H); ${ }^{13} \mathrm{C}$ NMR: (75 MHz, $\left.\mathrm{CDCl}_{3}\right): \delta$ 196.7, 137.8, 135.2, 134.7, 132.4, 132.2, 131.8, 130.1, 129.4, 128.30, 128.29, 128.26,127.8, 126.7, 125.7; MS (EI): 
$233\left(\mathrm{M}+1^{+}, 18\right), 232\left(\mathrm{M}^{+}, 92\right), 231$ (20), 155 (100), 127 (65), 105 (34), 77 (46); IR (KBr): $3055,1658,1270,1116,819,754,697 \mathrm{~cm}^{-1}$.

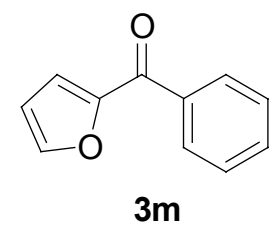

2-Benzoylfuran (3m) $)^{15}:{ }^{1} \mathrm{H}$ NMR (300 MHz, $\left.\mathrm{CDCl}_{3}\right): \delta \quad 7.99-7.96(\mathrm{~m}, 2 \mathrm{H}), 7.72(\mathrm{~s}$, $1 \mathrm{H}), 7.60-7.57(\mathrm{~m}, 1 \mathrm{H}), 7.53-7.48(\mathrm{~m}, 2 \mathrm{H}), 7.26-7.23(\mathrm{~m}, 1 \mathrm{H}), 6.61-6.59(\mathrm{~m}, 1 \mathrm{H}) .{ }^{13} \mathrm{C}$ NMR: $\left(75 \mathrm{MHz}, \mathrm{CDCl}_{3}\right): \quad \delta \quad 182.5,152.1,147.1,137.1,132.5,129.2,128.3,120.6$, 112.1.

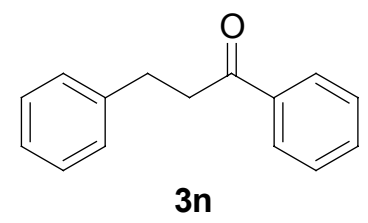

1,3-Diphenyl-1-propanone (3n) ${ }^{16}:{ }^{1} \mathrm{H}$ NMR $\left(300 \mathrm{MHz}, \mathrm{CDCl}_{3}\right): \delta 3.07(\mathrm{t}, J=8.0 \mathrm{~Hz}$, 2H), $3.30(\mathrm{t}, J=8.0 \mathrm{~Hz}, 2 \mathrm{H}), 7.18-7.32(\mathrm{~m}, 5 \mathrm{H}), 7.42-7.47$ (m, 2H), 7.55 (t, $J=7.8 \mathrm{~Hz}$, 1H), 7.95 (d, $J=7.8 \mathrm{~Hz}, 2 \mathrm{H}) ;{ }^{13} \mathrm{C}$ NMR: $\left(75 \mathrm{MHz}, \mathrm{CDCl}_{3}\right)$ : $\delta \quad 30.1,40.4,126.1,128.0$, 128.4, 128.5, 128.6, 133.1, 136.8, 141.2, 199.2; MS (EI): 211(M+1 $\left.{ }^{+}, 8\right), 210\left(\mathrm{M}^{+}, 46\right)$, 131 (1), 106 (9), 105 (100), 103 (5), 91 (11), 79 (3), 78 (6), 77 (396), 65 (41), 51 (9); IR (KBr): 3062, 3026, 1682, 1596, 1448, 1209, 973, 744, $689 \mathrm{~cm}^{-1}$.

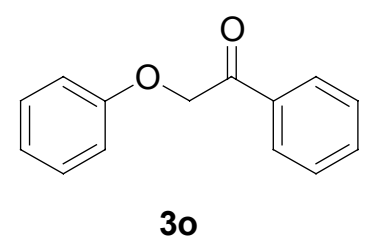

2-Phenoxyacetophenone (3o) ${ }^{17}:{ }^{1} \mathrm{H}$ NMR (300 MHz, $\left.\mathrm{CDCl}_{3}\right): \delta \quad 5.26(\mathrm{~s}, 2 \mathrm{H}), 6.92-7.00$ $(\mathrm{m}, 3 \mathrm{H}), 7.28(\mathrm{~m}, 2 \mathrm{H}), 7.46-7.51(\mathrm{~m}, 2 \mathrm{H}), 7.60(\mathrm{~m}, 1 \mathrm{H}), 7.97-8.00(\mathrm{~m}, 2 \mathrm{H}) ;{ }^{13} \mathrm{C} \mathrm{NMR}$ $\left(75 \mathrm{MHz}, \mathrm{CDCl}_{3}\right): \quad \delta \quad 194.5,157.9,134.5,133.8,129.5,128.8,128.1,121.6,114.7,70.7$; 
MS (EI): $213\left(\mathrm{M}+1^{+}, 6\right), 212(\mathrm{M}, 36), 106$ (8), 105 (100), 91 (10), 77 (90), 65 (15), 51 (41); IR (KBr): 3066, 1708, 1600, 1581, 1500, 1449, 1228, $1094 \mathrm{~cm}^{-1}$.

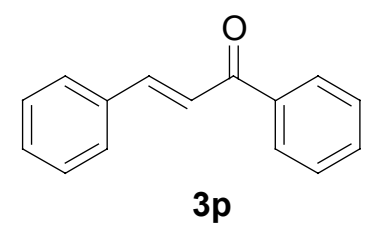

trans-Chalcon (3p) ${ }^{18}:{ }^{1} \mathrm{H}$ NMR $\left(300 \mathrm{MHz}, \mathrm{CDCl}_{3}\right): \delta 8.02 \quad(\mathrm{~d}, J=7.0 \mathrm{~Hz}, 2 \mathrm{H})$, $7.81(\mathrm{~d}, J=15.9 \mathrm{~Hz}, 1 \mathrm{H}), 7.63(\mathrm{~m}, 2 \mathrm{H}), 7.52$ (m, 4H), 7.40 (m, 3H); ${ }^{13} \mathrm{C}$ NMR: $(75 \mathrm{MHz}$, $\left.\mathrm{CDCl}_{3}\right): \delta$ 190.6, 144.8, 138.2, 134.8, 132.8, 130.6, 128.9, 128.6, 128.5, 128.4, 122.0; MS (EI): 209 (M+1 $\left.{ }^{+}, 12.27\right), 208$ (M+1, 80), 207 (100), 131 (23), 105 (17), 103 (23), 77 (51), 51 (23); IR (KBr): 3060, 1762, 1662, 1605, 1575, 1496, 1448, 978, 746, $689 \mathrm{~cm}^{-1}$.

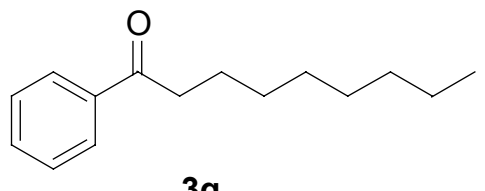

$3 q$

1-Phenyl-1-nonanone (3q) ${ }^{19}:{ }^{1} \mathrm{H}$ NMR $\left(300 \mathrm{MHz}, \mathrm{CDCl}_{3}\right): \delta \quad 7.94-7.98(\mathrm{~d}, J=7.1 \mathrm{~Hz}$, 2H), 7.52-7.58 (m, 1H), 7.42-7.48 (t, $J=7.1 \mathrm{~Hz}, 2 \mathrm{H}), 2.96(\mathrm{t}, J=7.8 \mathrm{~Hz}, 2 \mathrm{H}), 1.74(\mathrm{~m}$, $2 \mathrm{H}), 1.13-1.40(\mathrm{~m}, 10 \mathrm{H}), 0.88(\mathrm{t}, J=7.2 \mathrm{~Hz}, 3 \mathrm{H}) ;{ }^{13} \mathrm{C} \mathrm{NMR}:\left(75 \mathrm{MHz}, \mathrm{CDCl}_{3}\right): \delta$ 200.6, 137.0, 132.8, 128.5, 128.0, 38.6, 31.8, 29.4, 29.3, 29.1, 24.3, 22.6, 14.1; MS (EI): $219\left(\mathrm{M}+1^{+}, 4.14\right), 218\left(\mathrm{M}^{+}, 11\right), 133$ (11), 120 (100), 105 (81), 77 (27), 51 (4); IR (KBr): $3063,2928,2856,1690,1689,1582,1450,1258,1217 \mathrm{~cm}^{-1}$.

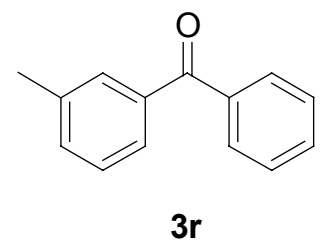

3-Methylbenzophenone (3r) ${ }^{20}:{ }^{1} \mathrm{H}$ NMR (300 MHz, $\mathrm{CDCl}_{3}$ ): $\delta$ 7.82-7.78 (m, $\left.2 \mathrm{H}\right)$, 7.63$7.56(\mathrm{~m}, 3 \mathrm{H}), 7.50-7.45$ (m, 2H), 7.40-7.33 (m, 2H), 2.42 (s, 3H); ${ }^{13} \mathrm{C}$ NMR (75 MHz, 
$\left.\mathrm{CDCl}_{3}\right): \delta 196.9,138.1,137.7,137.6,133.2,132.3,130.4,130.0,128.2,128.0,127.3$, 21.3; MS (EI) (\%): $197\left(\mathrm{M}+1^{+}, 27\right), 196\left(\mathrm{M}^{+}, 82\right), 119$ (100), 105 (64), 91 (43), 77 (44), 65 (24), 51 (22), 41 (2); IR: 1661, 1598, 1318, 1212, 958, 778, 720, $705 \mathrm{~cm}^{-1}$.

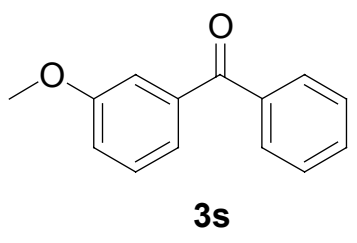

3-Methoxybenzophenone (3s) ${ }^{21}:{ }^{1} \mathrm{H}$ NMR $\left(300 \mathrm{MHz}, \mathrm{CDCl}_{3}\right): \delta \quad 7.82-7.79(\mathrm{~m}, 2 \mathrm{H})$, 7.59-7.56(m, 1H), 7.50-7.45 (m, 2H), 7.38-7.34 (t, 3H), 7.15-7.11 (m, 1H), 3.85 (s, 3H); ${ }^{13} \mathrm{C}$ NMR $\left(75 \mathrm{MHz}, \mathrm{CDCl}_{3}\right)$ : $\delta \quad 196.5,159.5,138.8,137.5,132.4,130.0,129.2,128.2$, 122.8, 118.8, 114.3, 55.4; MS (EI): $213\left(\mathrm{M}+1^{+}, 18\right), 212\left(\mathrm{M}^{+}, 100\right), 135$ (88), 107 (23), 105 (83), 92 (18), 77 (72), 64 (11), 51 (17); IR (KBr) 1660, 1597, 1580, 1450, 1430, $1283,1047,826,724,704 \mathrm{~cm}^{-1}$.

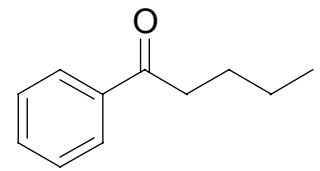

$3 t$

Pentanophenone (3t) $)^{22}:{ }^{1} \mathrm{H}$ NMR $\left(300 \mathrm{MHz}, \mathrm{CDCl}_{3}\right): \delta \quad 7.96(\mathrm{~d}, J=7.1 \mathrm{~Hz}, 2 \mathrm{H}), 7.57-$ $7.52(\mathrm{~m}, 1 \mathrm{H}), 7.45(\mathrm{t}, J=7.1 \mathrm{~Hz}, 2 \mathrm{H}), 2.96(\mathrm{t}, J=7.8 \mathrm{~Hz}, 2 \mathrm{H}), 1.72(\mathrm{~m}, 2 \mathrm{H}), 1.41(\mathrm{~m}$, 2H), 0.95 (t, $J=7.2 \mathrm{~Hz}, 3 \mathrm{H}) ;{ }^{13} \mathrm{C}$ NMR: $\left(75 \mathrm{MHz}, \mathrm{CDCl}_{3}\right)$ : $\delta \quad 200.5,137.0,132.8$, 128.4, 127.9, 38.2, 26.4,22.4, 13.9; MS (EI): $163\left(\mathrm{M}+1^{+}, 2\right), 162\left(\mathrm{M}^{+}, 14\right), 147$ (1), 133 (5), 120 (61), 105 (100), 85 (1), 77 (41), 57 (2), 51 (10), 43 (2), 41 (3); IR (KBr):3064, $2960,2874,1687,1598,1450,1267,1209,691 \mathrm{~cm}^{-1}$.

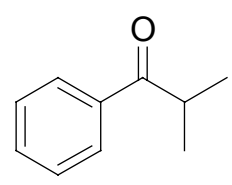

$3 \mathrm{u}$ 
Isobutyrophenone (3u) ${ }^{23}:{ }^{1} \mathrm{H} \mathrm{NMR}\left(300 \mathrm{MHz}, \mathrm{CDCl}_{3}\right): \delta 7.63(\mathrm{~d}, J=7.1 \mathrm{~Hz}, 2 \mathrm{H})$, 7.58-7.53 (m, 1H), 7.47 (t, $J=7.1 \mathrm{~Hz}, 2 \mathrm{H}), 3.57(\mathrm{~m}, J=6.6 \mathrm{~Hz}, 1 \mathrm{H}), 1.22(\mathrm{~d}, J=6.6 \mathrm{~Hz}$, $6 \mathrm{H}) ;{ }^{13} \mathrm{C} \mathrm{NMR}:\left(75 \mathrm{MHz}, \mathrm{CDCl}_{3}\right): \delta \quad 204.5,136.2,132.8,128.6,128.3,35.3,19.1$; MS (EI): $148\left(\mathrm{M}+1^{+}, 11.16\right), 147\left(\mathrm{M}^{+}, 39\right), 105$ (82), 77 (97), 71 (8), 51 (57), 43 (85), 41 (100); IR (KBr): 3063, 2972, 2874, 1685, 1467, 1448, 1384, 1225, 980, $703 \mathrm{~cm}^{-1}$.

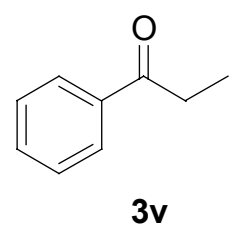

Propiophenone (3v) ${ }^{24}:{ }^{1} \mathrm{H}$ NMR (300 MHz, $\left.\mathrm{CDCl}_{3}\right): \delta \quad 7.97$ (d, $\left.J=7.1 \mathrm{~Hz}, 2 \mathrm{H}\right), 7.58$ $7.52(\mathrm{~m}, 1 \mathrm{H}), 7.45$ (t, $J=7.1 \mathrm{~Hz}, 2 \mathrm{H}), 3.01$ (q, $J=6.9 \mathrm{~Hz}, 2 \mathrm{H}), 1.24(\mathrm{t}, J=6.9 \mathrm{~Hz}, 3 \mathrm{H})$; ${ }^{13} \mathrm{C} \mathrm{NMR}:\left(75 \mathrm{MHz}, \mathrm{CDCl}_{3}\right)$ : $\delta \quad 200.8,136.9,132.8,128.5,127.9,31.8,8.2$; $\mathrm{MS}(\mathrm{EI})$ : $135\left(\mathrm{M}+1^{+}, 3\right), 134\left(\mathrm{M}^{+}, 21\right), 105$ (100), 77 (54), 71 (10), 57 (16), 51 (19), 43 (11), 41 (7); IR (KBr): 2980, 2940, 1689, 1598, 1450, 1221, 952, 746, $691 \mathrm{~cm}^{-1}$.

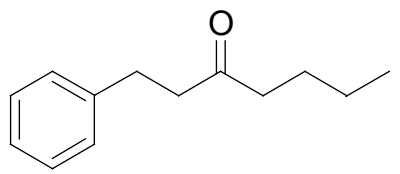

$3 w$

1-phenyl-heptan-3-one (3w) ${ }^{25}{ }^{1} \mathrm{H}$ NMR (300 MHz, $\left.\mathrm{CDCl}_{3}\right)$ : $\delta \quad 7.30-7.14(\mathrm{~m}, 5 \mathrm{H}), 2.86$ (t, $J=7.8 \mathrm{~Hz}, 2 \mathrm{H}), 2.70(\mathrm{t}, J=7.8 \mathrm{~Hz}, 2 \mathrm{H}), 2.36(\mathrm{t}, J=7.5 \mathrm{~Hz}, 2 \mathrm{H}), 1.53(\mathrm{~m}, J=7.5 \mathrm{~Hz}$, 2H), $1.27(\mathrm{~m}, J=7.5 \mathrm{~Hz}, 2 \mathrm{H}), 0.88(\mathrm{t}, J=7.5 \mathrm{~Hz}, 3 \mathrm{H}) ;{ }^{13} \mathrm{C} \mathrm{NMR}:\left(75 \mathrm{MHz}, \mathrm{CDCl}_{3}\right): \delta$ 210.2, 141.1, 128.4, 128.2, 126.2, 44.1, 42.6, 29.7, 25.8, 22.2, 13.7; MS (GC-EI): 190 $\left(\mathrm{M}^{+}, 40\right), 172$ (14), 161 (3), 148 (39), 130 (51), 115 (8), 105 (100), 91 (90), 77 (28), 57 (43).

\section{References:}


1. Armarego, W. L. F.; Perrin, D. D. Purification of Laboratory Chemicals $4^{\text {th }}$ Ed. Butterworth-Heinemann: Oxford, 1997.

2. Kim, J. and Jang, D. O. Synth. Commun. 2001, 31, 395.

3. Coulson, D. R. Inorg. Synth. 1972, 13, 121.

4. (a) Hwang, Je Pil; Prakash, G. K. Surya; Olah, George A. Tetrahedron 2000, 56, 7199. (b) Wu, Tse-Chong; Xiong, Heping; Rieke, R. D. J. Org. Chem. 1990, 55, 5045 .

5. (a) Goossen, L. J.; Ghosh, K. Eur. J. Org. Chem. 2002, 19, 3254. (b) Taber, D. F.; Sethuraman, M. R. J. Org. Chem. 2000, 65, 254.

6. Kakino, R.; Narahashi, H.; Shimizu, I.; Yamamoto, A. Bull. Chem. Soc. Jpn. 2002, 75, 1333.

7. (a) Nilsson, P.; Larhed, M.; Hallberg, A. J. Am. Chem. Soc. 2001; 123, 8217. (b) Blanco, F. E.; Harris, F. L. J. Org. Chem. 1977, 42, 868-871.

8. Moorthy, J. N.; Monahan, S. L.; Sunoj, R. B.; Chandrasekhar, J.; Bohne, C. J. Am. Chem. Soc. 1999, 121, 3093 .

9. Sakurai, H.; Imamoto, Y.; Hirao, T. Chem. Lett. 2002, 44.

10. (a) Singh, R. P.; Kamble, R. M.; Chandra, K. L.; Saravanan, P.; Singh, V. K. Tetrahedron, 2001, 57, 241. (b) Parham, W. E.; Bradsher, C. K.; Edgar, K. J. J. Org. Chem. 1981, 46, 1057. (c) Bomben, A.; Marques, C. A.; Selva, M.; Tundo, P. Synthesis, 1996, 1109-1114.

11. (a) Kalena, G. P.; Jadhav, S. M.; Banerji, A. Molecules, 2000, 5, 240. (b) Wagner, P. J.; Meador, M. A.; Park, B.-S. J. Am. Chem. Soc. 1990, 112, 5199. (c) Tomioka, H.; Nakanishi, K.; Izawa, Y. J. Chem. Soc. Perkin Trans. 1, 1991, 465.

12. (a) Rieke, R. D.; Klein, W. R.; Wu, T.-C. J. Org. Chem. 1993, 58, 2492. (b) Katritzky, A. R.; Rahimi-Rastgoo, S.; Sabongi, G. J.; Fischer, G. W. J. Chem. Soc. Perkin Trans.1, 1980, 362. (c) Kakino, R.; Yasumi, S.; Shimizu, I.; Yamamoto, A. Bull. Chem. Soc. Jpn. 2002, 75, 137.

13. (a) Suzuki, H.; Murashima, T. J. Chem. Soc. Perkin Trans.1, 1994; 903. (b) Andrus, M. B.; Ma, Y.; Zang, Y.; Song, C. Tetrahedron Lett. 2002, 43, 9137. (c) Frahm, A. W.; Hambloch, H. F. Org. Magn. Reson. 1980, 14, 444.

14. (a) Kim, S. -H.; Rieke, R. D. Synth. Commun. 1998, 28, 1065. (b) Kang, S.-K.; 
Ryu, H. -C.; Lee, S.-W. Synth. Commun. 2001, 31, 1027.

15. Kang, S.-K.; Ryu, H.-C.; Lee, S.-W. J. Organomet. Chem. 2000, 610, 38.

16. Gossen, L. J.; Ghosh, K. Angew. Chem. Int. Ed. 2001, 40, 3458.

17. Kandanarachchi, P. H.; Autrey, T.; Franz, J. A. J. Org. Chem. 2002, 67, 7937.

18. Cave, G. W. V.; Raston, C. L. J. Chem. Soc. Perkin Trans. 1, 2001, 3258.

19. Jensen, A. E.; Knochel, P. J. Org. Chem. 2002, 67, 79.

20. Goossen, L. J.; Ghosh, K. Eur. J. Org. Chem. 2002, 19, 3254.

21. Morley, J. A.; Woolsey, N. F. J. Org. Chem. 1992, 57, 6487.

22. (a)Yamamoto, H. M.; Sakurai, H.; Narasaka, K. Bull. Chem. Soc. Jpn. 1996, 69, 157. (b) Kim, S.; Lee, J. I. J. Org. Chem. 1983, 48, 26080.

23. Blay, G.; Fernandez, I.; Formentin, P.; Monje, B.; Pedro, J. R.; Ruiz, R. Tetrahedron 2001, 57, 1075.

24. Huang, Z.-Z.; Tang, Y. J. Org. Chem. 2002, 67, 5320.

25. Trost, B. M.; Kulawiec, R. J. J. Am. Chem. Soc. 1993, 115, 2027. 
<smiles>O=C(c1ccccc1)c1ccc(C(F)(F)F)cc1</smiles>

SIOC-OMCMercuryVX-300F19 Sensitivity TestSample: 0.05\% Trifluorotoluene2002

F1: 282.286 F2: 300.029 SW1: 84746 PW: 5.1 usec

PD: $1.5 \mathrm{sec}$
OF1: -22384.9

NA: 4

USER: -- DATE: Jul 82003 PTS1d: 65536

WinNuts - \$wdh-5-65-1-1-f.fid 


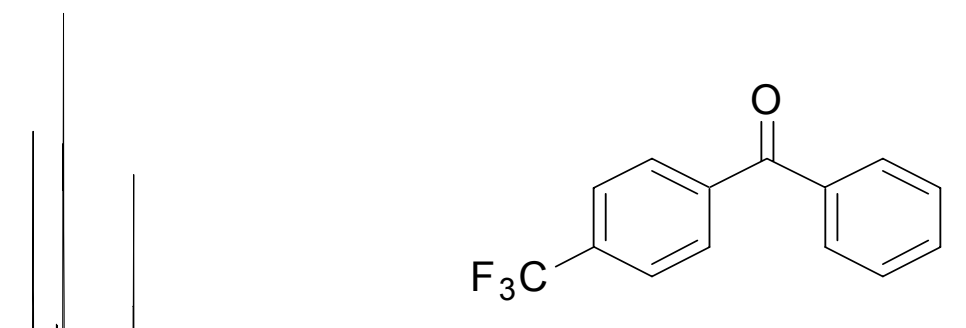

2.020042 .06

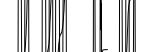

1.00

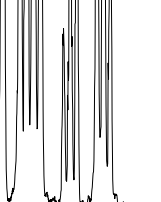

8

6

4

2

0 PPM

txf-7-14

F1: 300.030

F2: 75.450

SW1: 5495

OF1: 1810.9

PW: 3.8 usec

PD: $1.0 \mathrm{sec}$

NA: 4

PTS1d: 16384

USER: -- DATE: Jul 82003

WinNuts - \$wdh-5-65-1-1-h.fid 


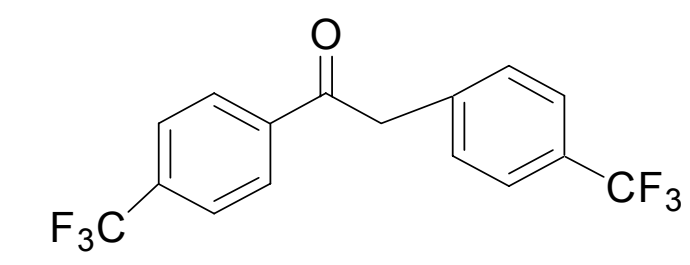

1ㅍำ 2.00

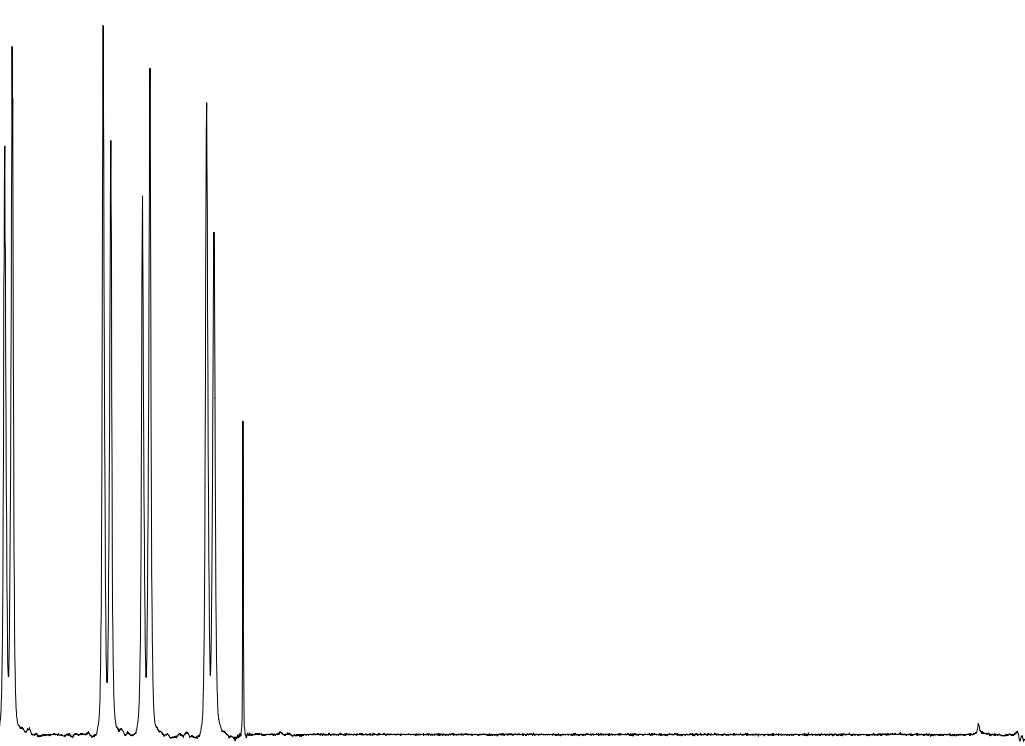

10

8

6

2

PPM

txf-7-14

F1: 300.030

F2: 75.450

SW1: 5495

4

USER: -- DATE: Jul 82003

EX: s2pul

PW: 3.8 usec

OF1: 1810.9

PTS1d: 16384

WinNuts - \$wdh-5-72-1-1-h.fid 

<smiles>O=C(Cc1ccc(C(F)(F)F)cc1)c1ccc(C(F)(F)F)cc1</smiles>

NA: 16 PTS1d: 65536 


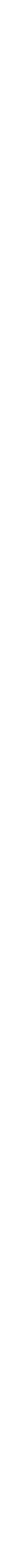


<smiles>O=C(Cc1ccc(C(F)(F)F)cc1)c1ccccc1</smiles>

Zgg-5-25-2aF1: 75.450

EX: s2pul
SW1: 18657

PW: 4.2 usec
100

OF1: 8290.2

NA: 44
LB: 0.0 PTS1d: 32768

USER: -- DATE: Apr 162003 WinNuts - \$wdh5-73-1C.fid 
<smiles>O=C(Cc1ccc(C(F)(F)F)cc1)c1ccccc1</smiles>

SIOC-OMCMercuryVX-300F19 Sensitivity TestSample: $0.05 \%$ Trifluorotoluene2002

F1: 282.286 F2: 300.029 SW1: 84746 PW: 5.1 usec

OF1: -22384.9

PD: $1.5 \mathrm{sec}$
NA: 16

LB: 0.0

USER: -- DATE: Jul 82003 PTS1d: 65536 
1.88

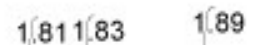

89

6
2

txf-7-14

F1: 300.030

F2: 75.450

SW1: 5495

PW: 3.8 usec

PD: $1.0 \mathrm{sec}$

OF1: 1810.9

4

NA: 4

PTS1d: 16384 
<smiles>COc1ccc(C(=O)Cc2ccc(C(F)(F)F)cc2)cc1</smiles>

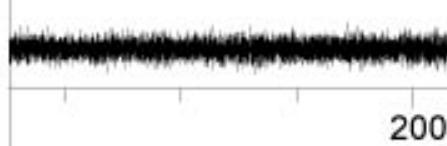

200
150
100

OF1: 8280.8

SW1: 18868

PW: 4.2 usec

NA: 124
50

0 PPM

$7 g g-7-73 \mathrm{c}$

F1: 75.450

F2: 300,029

LB: 0,0

USER: -- DATE: Jul 82003

WinNuts - \$wdh-5-74-1-1-c, fid 
<smiles>COc1ccc(C(=O)Cc2ccc(C(F)(F)F)cc2)cc1</smiles>

SIOC-OMCMercuryVX-300F19 Sensitivity TestSample: $0.05 \%$ Trifluorotoluene2002

F1: 282.286 F2: 300.029 SW1: 84746 PW: 5.1 usec

OF1: -22384.9

PD: $1.5 \mathrm{sec}$
NA:

LB: 0.0

USER: -- DATE: Jul 82003 PTS1d: 65536 


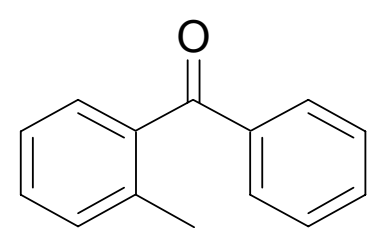

5.86

1.75

0.95 


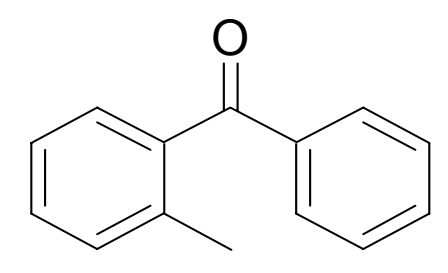

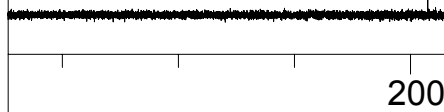

200
150
100

SW1: 18868

PW: 4.2 usec

PD: $1.0 \mathrm{sec}$

OF1: 8271.9

NA: 32
50

F1: 75.450

EX: s2pul

LB: 0.0

PTS1d: 65536

ISER:- DATE: May 292003 WinNuts - \$wdh-6-25-1-2c.fid 
<smiles>Cc1ccc(C(=O)c2ccccc2)cc1</smiles>

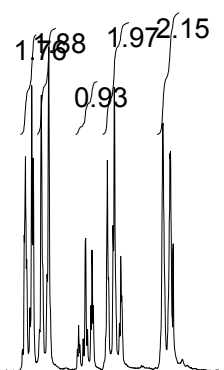

OPPM STANDARD 1H OBSERVE F1: 300.030

USER: -- DATE: May 222003 
<smiles>COc1ccc(C(=O)c2ccccc2)cc1</smiles>

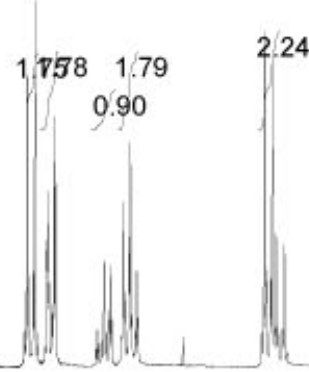

6

4
2

OF1: 1803.0

PD: $1.0 \mathrm{sec}$

NA: 6
LB: 0.0
PTS1d: 16384
PW: 3.7

F2: 75.450

PW: 3.7 usec
USER: -- DATE: May 222003

WinNuts - \$wdh-6-27-1. fid 


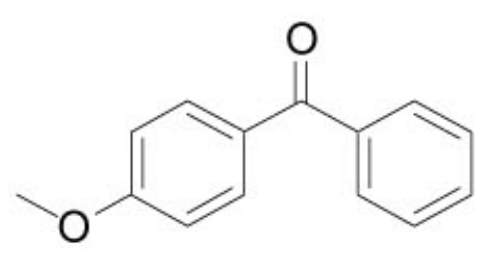

OFI: 8272.2

NA: 32

USER: -- DATE: May 222003 PTSId: 65536

WinNuts - \$wdh-6-27-1c.fid 
<smiles>COc1ccccc1C(=O)c1ccccc1</smiles>

4.74

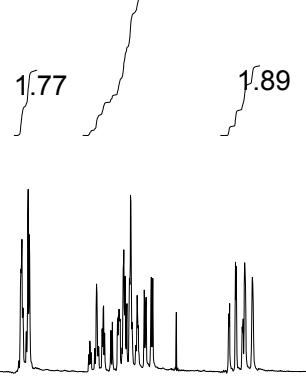

3.00

USER: -- DATE: May 282003 
<smiles>COc1ccccc1C(=O)c1ccccc1</smiles>

(innen, 
<smiles>O=C(c1ccccc1)c1ccc2ccccc2c1</smiles>
F2: 75.450

SW1: 6667

PW: 3.7 usec
6

4 OF1: 1797.6 PD: $1.0 \mathrm{sec}$
NA: 4
2 PTS1d: 16384 USER: -- DATE: May 282003

WinNuts - \$wdh-6-29-1.fid 


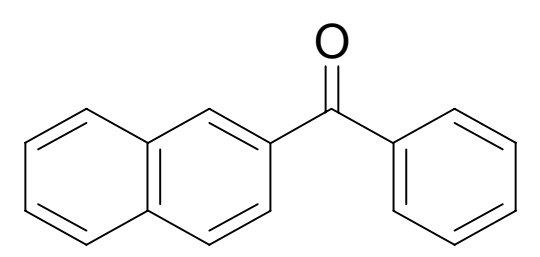

\section{Whinim.}




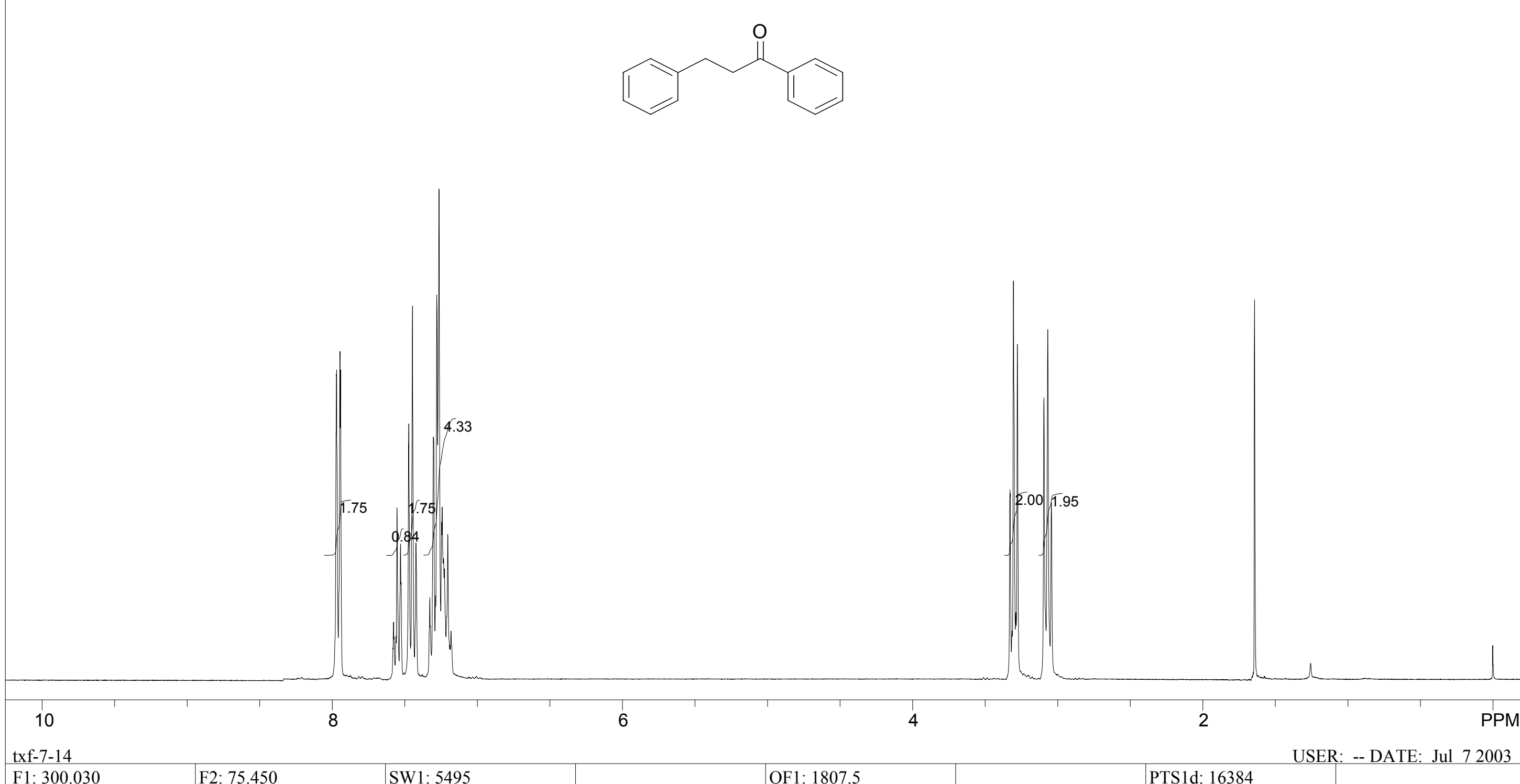





$$
\text { aro }
$$


<smiles>O=C(c1ccccc1)c1ccc([N+](=O)[O-])cc1</smiles>

USER: -- DATE: May 282003 


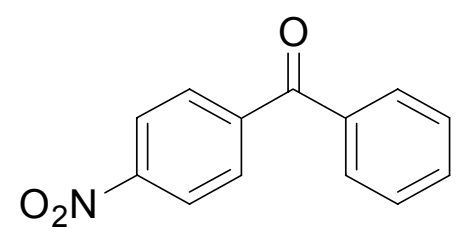

PPM

zgg-7-19c

F1: 75.450

EX: s2pul
SW1: 18868

PW: 4.2 usec
OF $1: 8278.0$

NA: 28
LB: 0.0 USER: -- DATE: May 282003 

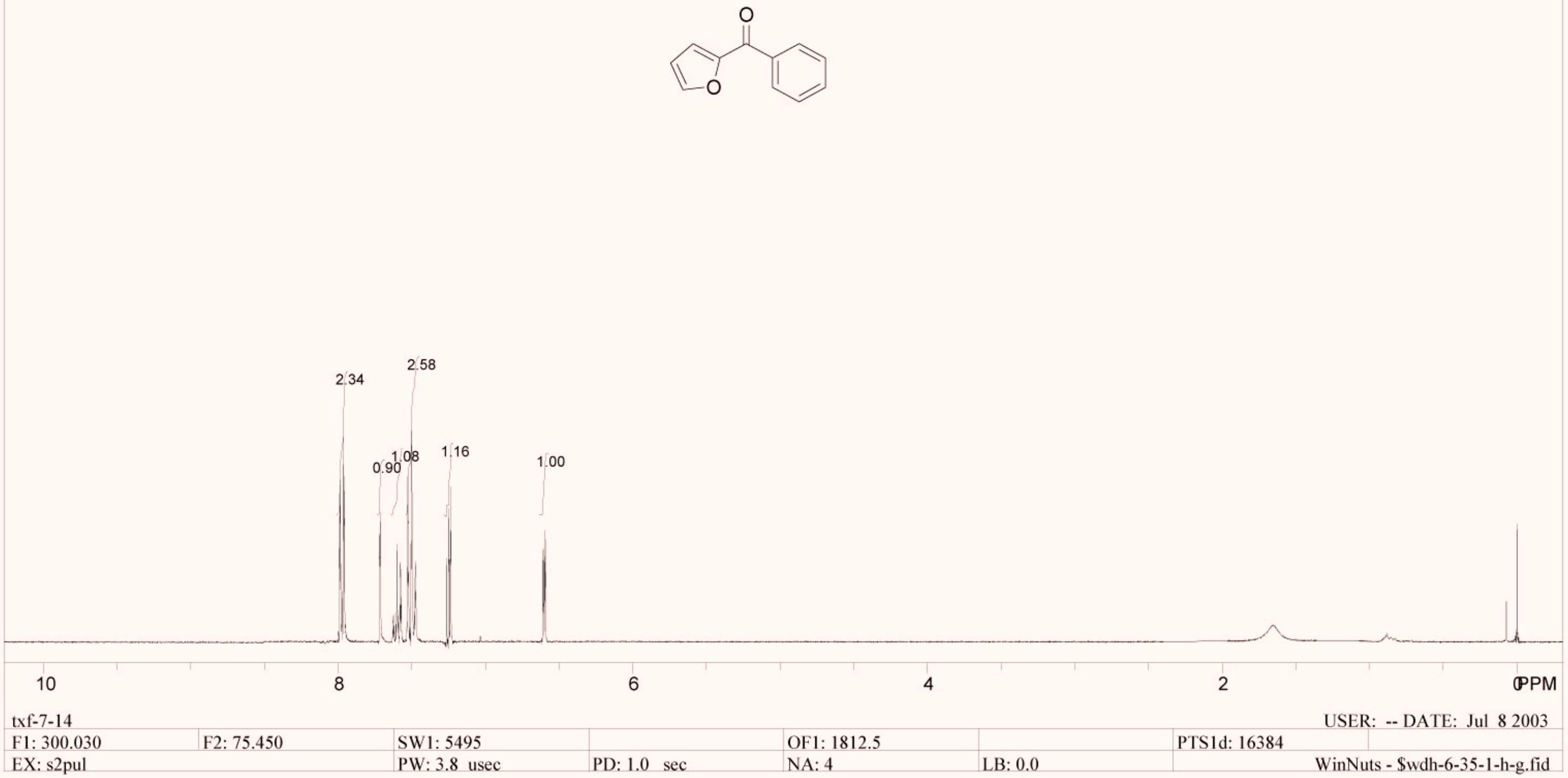



$$
\text { oro }
$$


<smiles>O=C(c1ccccc1)c1ccc(Cl)cc1</smiles>

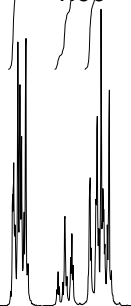

10

wf-6-19-3 F1: 300.030 EX: s2pul 


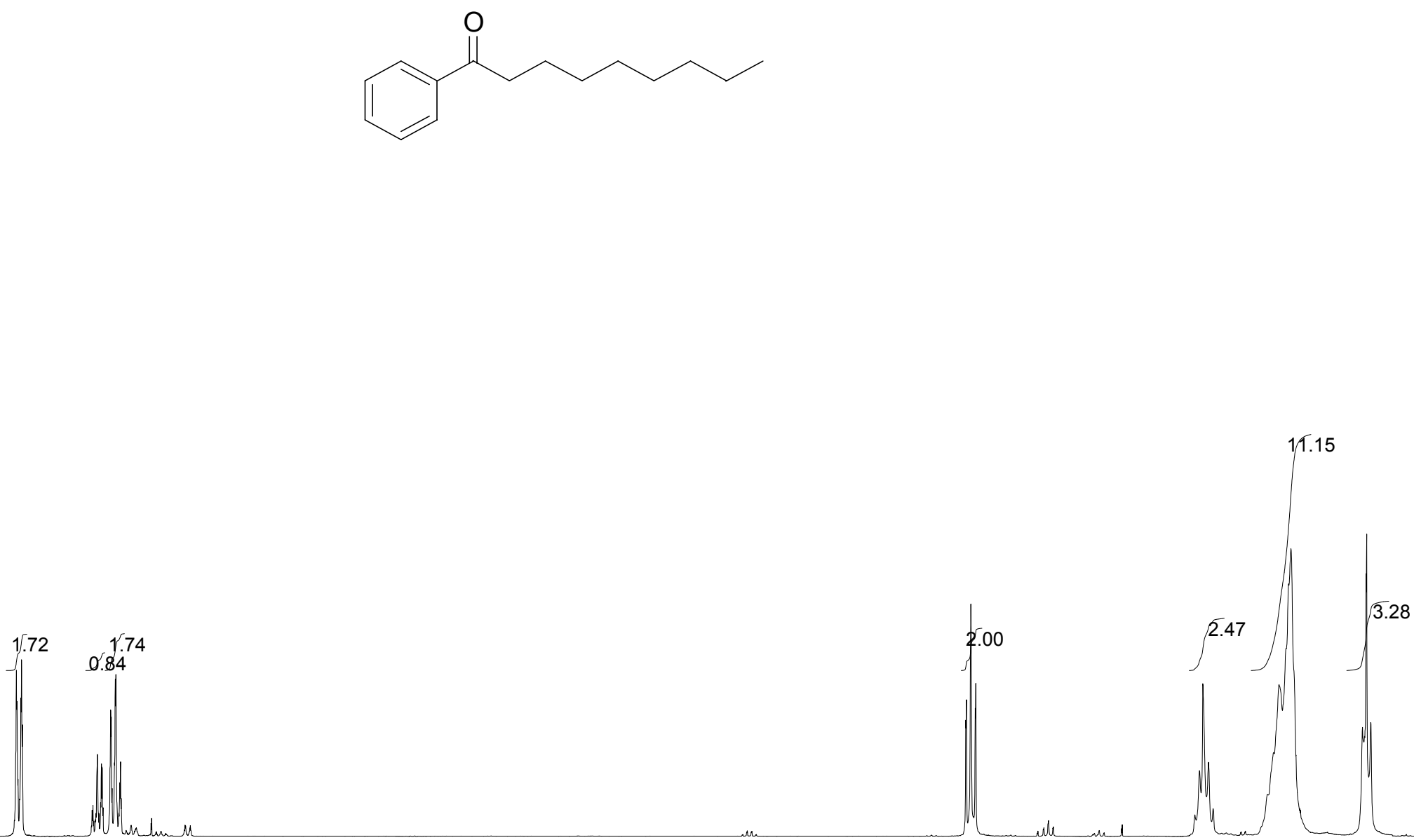

txf-7-14

F1: 300.030

F2: 75.450

SW1: 5495

PW: 3.8 usec

OF1: 1812.9

PD: $1.0 \mathrm{sec}$
PTS1d: 16384

USER: -- DATE: Jul 72003 


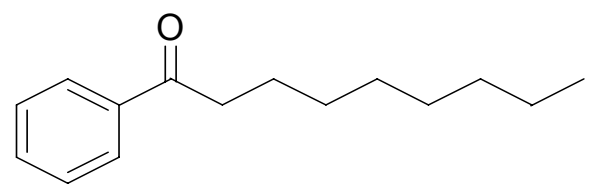

zgg-7-73c

F1: 75.450

EX: s2pul
SW1: 18868

PW: 4.2 usec
OF1: 8278.7

NA: 20

LB: 0.0

USER: -- DATE: Jul 72003 PTS1d: 65536

WinNuts - \$wdh-6-49-1-c.fid 


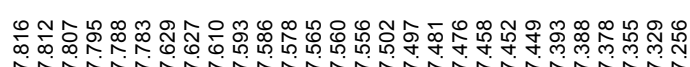

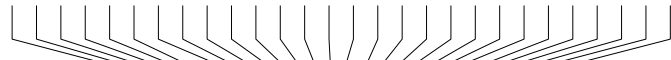

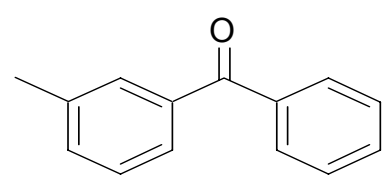

2.74

$1.83 \quad 1.96$

$1.83 \quad 1.96$

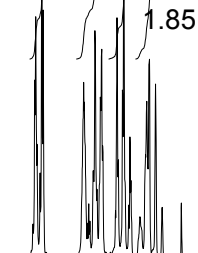




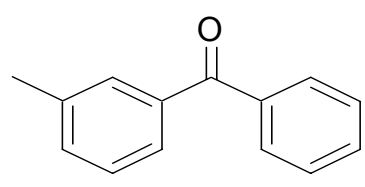


wdh-6-81-1

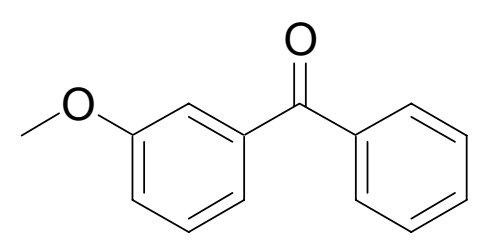

2.70

$1.69 \quad 1.81$

$0.87 \quad 0.89$

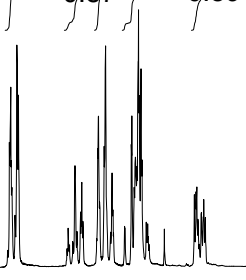

4

F2: 75.450

gmj-9-70-2

F1: 300.030

EX: s2pul
SW1: 4803

PW: 3.7 usec
OF1: 1800.8

PD: $1.0 \mathrm{sec}$

NA: 22

LB: 0.0

2

PTS1d: 16384

USER: -- DATE: Aug 262003

WinNuts - \$wdh-6-81-1.fid 


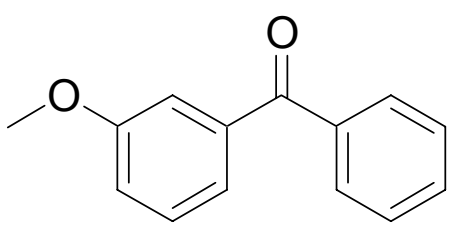

PPM

gmj-9-70-2-c F1: 75.450

EX: s2pul
SW1: 18868

PW: 4.2 usec
OF1: 8278.2

NA: 44

LB: 0.0 PTS1d: 65536

USER: -- DATE: Aug 262003 PD: $1.0 \mathrm{sec}$

WinNuts - \$wdh-6-81-1-C.fid 


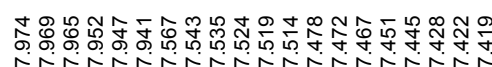

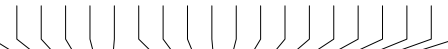

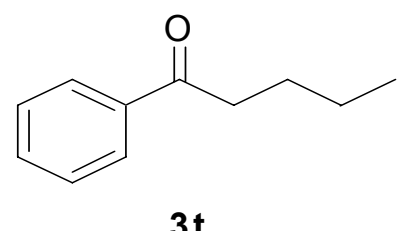

$3 t$

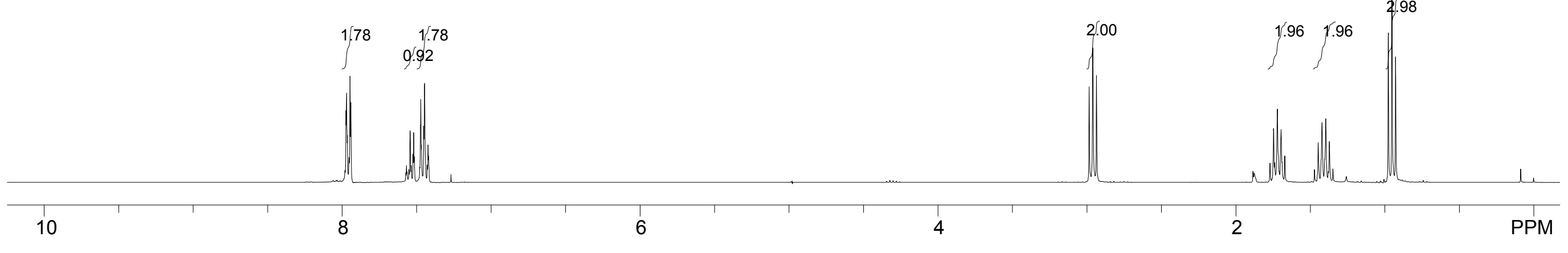


wdh-6-73-1

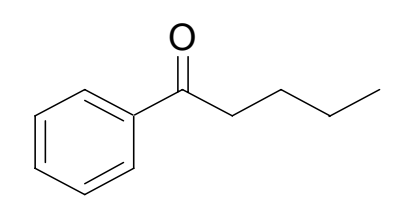




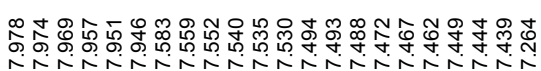

W

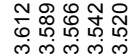

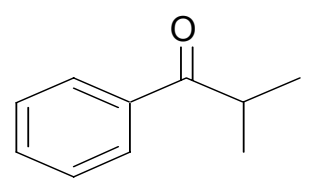

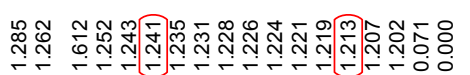

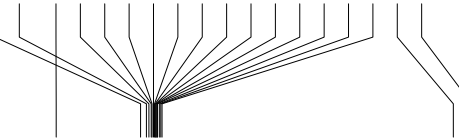


단ำ

인

ำ

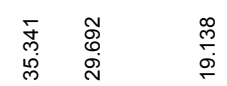

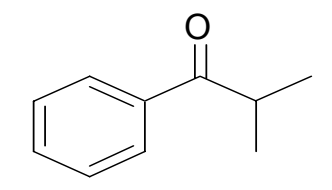




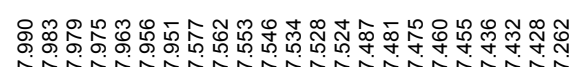

保

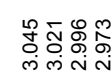

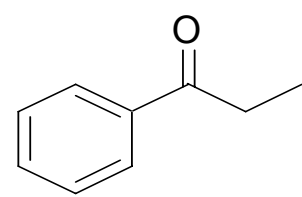

高

:̊ำ

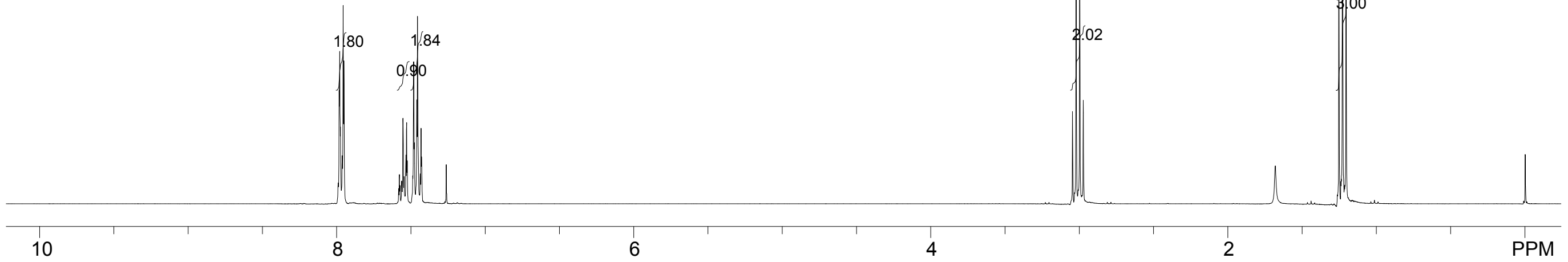




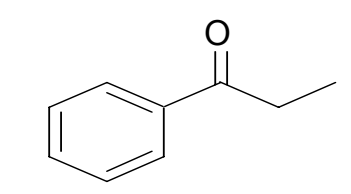




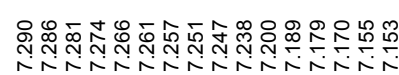

wdh-6-97-1

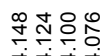

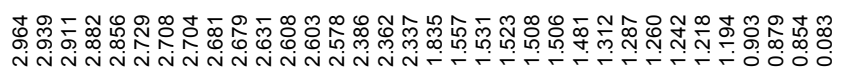

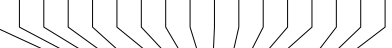

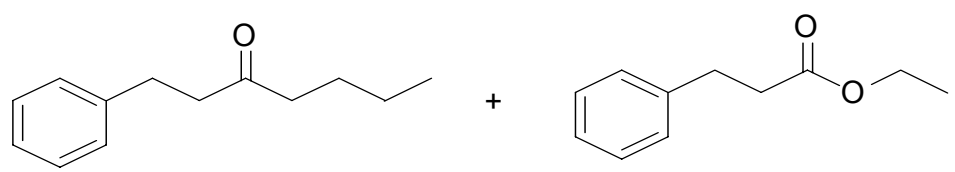

4.18

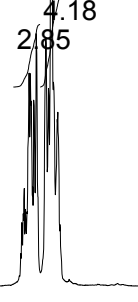




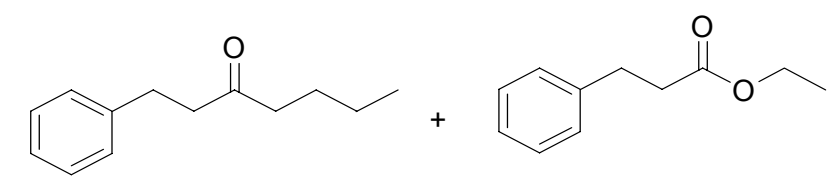

wdh-6-97-1-C

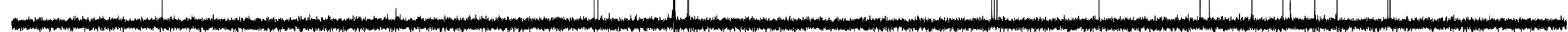

